Geometry $\&$ Topology

Volume 2 (1998) 1-10

Published: 16 January 1998

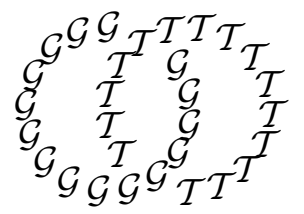

\title{
Einstein metrics and smooth structures
}

\author{
D KoTschick \\ Mathematisches Institut \\ Universität Basel \\ Rheinsprung 21 \\ 4051 Basel, Switzerland \\ Email: dieter@math-lab.unibas.ch
}

\begin{abstract}
We prove that there are infinitely many pairs of homeomorphic non-diffeomorphic smooth 4-manifolds, such that in each pair one manifold admits an Einstein metric and the other does not. We also show that there are closed 4-manifolds with two smooth structures which admit Einstein metrics with opposite signs of the scalar curvature.
\end{abstract}

AMS Classification numbers Primary: 57R55, 57R57, 53C25

Secondary: $14 \mathrm{~J} 29$

Keywords: Einstein metric, smooth structure, four-manifold

Proposed: Peter Kronheimer

Seconded: Ronald Stern, Gang Tian
Received: 8 September 1997

Revised: 14 January 1998

Copyright Geometry and Topology 
In dimensions strictly smaller than four Einstein metrics have constant curvature and are therefore rare. In dimension four Einstein metrics of non-constant curvature exist, but it is still the case that existence of such a metric imposes non-trivial restrictions on the underlying manifold ${ }^{1}$. For closed orientable Einstein 4-manifolds $X$ the Euler characteristic has to be non-negative, and, furthermore, the Hitchin-Thorpe inequality

$$
e(X) \geq \frac{3}{2}|\sigma(X)|
$$

must hold, where $e$ denotes the Euler characteristic and $\sigma$ the signature. This condition is very crude, and is certainly homotopy invariant, as are the restrictions coming from Gromov's notion of simplicial volume [11], and from the existence of maps of non-zero degree to hyperbolic manifolds [21].

Our aim in this note is to discuss existence and non-existence of Einstein metrics as a property of the smooth structure. We shall exhibit infinitely many pairs of homeomorphic non-diffeomorphic smooth 4-manifolds, such that in each pair one manifold admits an Einstein metric and the other does not. This shows for the first time that the smooth structures of 4-manifolds form definite obstructions to the existence of an Einstein metric.

An isolated example of such a pair can be obtained as follows. Hitchin [12] showed that Einstein manifolds for which (1) is an equality are either flat or quotients of a $K 3$ surface with a Calabi-Yau metric. Thus, the existence of smooth manifolds homeomorphic but not diffeomorphic to the $K 3$ surface, which is known from Donaldson theory [10] and also follows easily from Seiberg-Witten theory, see for example $[7,15]$, shows that by changing only the differentiable structure one can pass from a manifold with an Einstein metric to one without. The point of our examples is that there are lots of them, and they do not arise from the borderline case of a non-existence result. They are in some sense generic.

We shall also discuss a conjecture concerning uniqueness of Einstein metrics on 4-manifolds which complements the discussion of existence. This too depends on a consideration of different smooth structures on a fixed topological manifold.

\section{Smooth structures as obstructions}

We shall use Seiberg-Witten invariants to show that certain smooth structures obstruct the existence of Einstein metrics, and refer the reader to $[24,7,15]$

\footnotetext{
${ }^{1}$ No such restrictions are known in higher dimensions.
} 
for the definitions and basic properties of the invariants. All manifolds in this section are closed, smooth, oriented 4-manifolds. For the sake of simplicity, we assume $b_{2}^{+}>1$ throughout, though this is not essential.

We shall need the following result concerning the behaviour of the invariants under connected summing with $\overline{\mathbb{C} P^{2}}$. This is usually referred to as a blowup formula.

Proposition $1.1([8,16])$ Let $\widetilde{P}(Y)$ be a $\operatorname{Spin}^{c}$-structure on $Y$, and $X=$ $Y \# \overline{\mathbb{C P}}^{2}$, with $E$ a generator of $H^{2}\left(\overline{\mathbb{C P}}^{2}, \mathbb{Z}\right)$. Then $X$ has a $\operatorname{Spin}^{c}$-structure $\widetilde{P}(X)$ with $c_{1}(\widetilde{P}(X))=c_{1}(\widetilde{P}(Y))+E$, such that the Seiberg-Witten invariants of $\widetilde{P}(Y)$ and of $\widetilde{P}(X)$ are equal (up to sign).

As the reflection in $E^{\perp}$ in the cohomology of $X$ is realised by a self-diffeomorphism, the naturality of the invariants shows that there is another $\operatorname{Spin}^{c}-$ structure with the same Seiberg-Witten invariant, up to sign, and with $c_{1}(\widetilde{P}(X))=c_{1}(\widetilde{P}(Y))-E$.

Using this, we can prove the following version of a theorem of LeBrun [18]:

Theorem 1.2 Let $Y$ be a manifold with a non-zero Seiberg-Witten invariant (of any degree), and $X=Y \# k \overline{\mathbb{C P}}^{2}$. If $k>\frac{2}{3}(2 e(Y)+3 \sigma(Y))$, then $X$ does not admit an Einstein metric.

Proof If $\widetilde{P}(X)$ has a non-zero Seiberg-Witten invariant, then for every Riemannian metric $g$ there must be a solution $(A, \phi)$ of the monopole equations. Denoting by $\hat{A}$ the connection induced by the $\operatorname{Spin}^{c}$-connection $A$ on the determinant bundle of the spinor bundle, we have

$$
\begin{aligned}
c_{1}^{2}(\widetilde{P}(X)) & =\frac{1}{4 \pi^{2}} \int_{X}\left(\left|F_{\hat{A}}^{+}\right|^{2}-\left|F_{\hat{A}}^{-}\right|^{2}\right) d \operatorname{vol}_{g} \leq \frac{1}{4 \pi^{2}} \int_{X}\left|F_{\hat{A}}^{+}\right|^{2} d \operatorname{vol}_{g} \\
& =\frac{1}{32 \pi^{2}} \int_{X}|\phi|^{4} d \operatorname{vol}_{g} \leq \frac{1}{32 \pi^{2}} \int_{X} s_{g}^{2} d \operatorname{vol}_{g},
\end{aligned}
$$

where $s_{g}$ denotes the scalar curvature of $g$.

Given any class $c \in H^{2}(X, \mathbb{R})$, denote by $c^{+}$the projection of $c$ into the subspace $\mathcal{H}_{+}^{2} \subset \mathcal{H}^{2}$ of $g$-self-dual harmonic forms along the subspace $\mathcal{H}_{-}^{2}$ of $g$-anti-self-dual harmonic forms. The argument above really proves

$$
\left(c_{1}(\widetilde{P}(X))^{+}\right)^{2} \leq \frac{1}{32 \pi^{2}} \int_{X} s_{g}^{2} d \operatorname{vol}_{g} .
$$


If $\widetilde{P}(Y)$ is a $\operatorname{Spin}^{c}$-structure on $Y$ with non-zero Seiberg-Witten invariant, then, by Proposition 1.1 and the subsequent remark, there are $\operatorname{Spin}^{c}$-structures $\widetilde{P}(X)$ on $X=Y \# k \overline{\mathbb{C P}}^{2}$ with non-zero Seiberg-Witten invariants and with

$$
c_{1}(\widetilde{P}(X))=c_{1}(\widetilde{P}(Y))+\sum_{i=1}^{k}(-1)^{\epsilon_{i}} E_{i}
$$

for any choice of the signs $(-1)^{\epsilon_{i}}$. Choose the signs so that

$$
(-1)^{\epsilon_{i}} E_{i}^{+} \cdot c_{1}(\widetilde{P}(Y))^{+} \geq 0 .
$$

Then

$$
\begin{aligned}
& \frac{1}{32 \pi^{2}} \int_{X} s_{g}^{2} d \operatorname{vol}_{g} \geq\left(c_{1}(\widetilde{P}(X))^{+}\right)^{2} \\
&=\left(c_{1}(\widetilde{P}(Y))^{+}\right)^{2}+2 \sum_{i=1}^{k}(-1)^{\epsilon_{i}} E_{i}^{+} \cdot c_{1}(\widetilde{P}(Y))^{+}+\left(\sum_{i=1}^{k}(-1)^{\epsilon_{i}} E_{i}^{+}\right)^{2} \\
& \geq\left(c_{1}(\widetilde{P}(Y))^{+}\right)^{2} \geq c_{1}(\widetilde{P}(Y))^{2} \geq 2 e(Y)+3 \sigma(Y) \\
& \quad=2(e(X)-k)+3(\sigma(X)+k)=2 e(X)+3 \sigma(X)+k
\end{aligned}
$$

where we have used the inequality $c_{1}(\widetilde{P}(Y))^{2} \geq 2 e(Y)+3 \sigma(Y)$ which is equivalent to the assertion that the moduli space associated with $\widetilde{P}(Y)$ has nonnegative dimension.

Thus, we have proved $\frac{1}{32 \pi^{2}} \int_{X} s_{g}^{2} d \operatorname{vol}_{g} \geq 2 e(X)+3 \sigma(X)+k$ for every metric $g$ on $X$.

Suppose now that $g$ is Einstein. Then the Chern-Weil integrals for the Euler characteristic and the signature of $X$ give

$$
\begin{aligned}
2 e(X)+3 \sigma(X) & =\frac{1}{4 \pi^{2}} \int_{X}\left(\frac{1}{24} s_{g}^{2}+2\left|W_{+}\right|^{2}\right) d \operatorname{vol}_{g} \\
& \geq \frac{1}{96 \pi^{2}} \int_{X} s_{g}^{2} d \operatorname{vol}_{g} \\
& \geq \frac{1}{3}(2 e(X)+3 \sigma(X)+k),
\end{aligned}
$$

where $W_{+}$denotes the self-dual part of the Weyl tensor of $g$. Therefore $k \leq$ $2(2 e(X)+3 \sigma(X))=2(2 e(Y)+3 \sigma(Y)-k)$, which implies $k \leq \frac{2}{3}(2 e(Y)+$ $3 \sigma(Y))$.

Theorem 1.2 was proved by LeBrun [18], who also discussed the borderline case $k=\frac{2}{3}(2 e(Y)+3 \sigma(Y))$, in the case where $Y$ is complex or symplectic. In that 
case the blown up manifold $X$ is also complex, respectively symplectic, so that Proposition 1.1 is not needed.

The following is the main result of this section, giving the examples mentioned in the introduction.

Theorem 1.3 There are infinitely many pairs $\left(X_{i}, Z_{i}\right)$ of simply connected closed oriented smooth 4-manifolds such that:

1) $X_{i}$ is homeomorphic to $Z_{i}$,

2) if $i \neq j$, then $X_{i}$ and $X_{j}$ are not homotopy equivalent,

3) $Z_{i}$ admits an Einstein metric but $X_{i}$ does not,

4) $e\left(X_{i}\right)>\frac{3}{2}\left|\sigma\left(X_{i}\right)\right|$.

Note that 3) implies in particular that $X_{i}$ and $Z_{i}$ are not diffeomorphic.

Proof We claim that there are simply connected minimal complex surfaces $Y_{i}, Z_{i}$ of general type such that if we take $X_{i}=Y_{i} \# k \overline{\mathbb{C} P^{2}}$, for a suitable $k$ with $k>\frac{2}{3}\left(2 e\left(Y_{i}\right)+3 \sigma\left(Y_{i}\right)\right)$, then the pairs $\left(X_{i}, Z_{i}\right)$ have all the desired properties. The last property, the strict Hitchin-Thorpe inequality, follows from the Noether and Miyaoka-Yau inequalities for $Z_{i}$, which, by the first property, has the same Euler characteristic and signature as $X_{i}$.

If we take $Z_{i}$ to have ample canonical bundle, then the results of Aubin and Yau on the Calabi conjecture show that $Z_{i}$ admits a Kähler-Einstein metric, compare [3]. On the other hand, $X_{i}$ does not admit any Einstein metric by Theorem 1.2.

The crucial issue then is to arrange that $Z_{i}$, with ample canonical bundle, is homeomorphic to the $k$-fold blowup of $Y_{i}$, with $k>\frac{2}{3}\left(2 e\left(Y_{i}\right)+3 \sigma\left(Y_{i}\right)\right)$. As $X_{i}$ will be automatically non-spin, $X_{i}$ and $Z_{i}$ will be homeomorphic by Freedman's classification [9] as soon as $Z_{i}$ is non-spin and has the same Euler characteristic and the same signature as $X_{i}$. One can find suitable surfaces using the known results on the geography of surfaces of general type, see [14] for a summary of the results.

To exhibit concrete examples, instead of working with the topological Euler characteristic and the signature, we shall use the first Chern number $c_{1}^{2}=$ $2 e+3 \sigma$ and the Euler characteristic of the structure sheaf $\chi=\frac{1}{4}(e+\sigma)$.

Under blowing up, $c_{1}^{2}$ drops by one and $\chi$ is constant. Thus, the Miyaoka-Yau inequality for $Y_{i}$ implies $c_{1}^{2}\left(X_{i}\right)<3 \chi\left(X_{i}\right)$. In fact, $c_{1}^{2}\left(X_{i}\right)$ will be smaller still, 
because simply connected surfaces $Y_{i}$ are not known to exist if we get too close to the Miyaoka-Yau line $c_{1}^{2}=9 \chi$.

The minimal surface $Z_{i}$ satisfies the same inequalities on its characteristic numbers as $X_{i}$. If the canonical bundle of $Z_{i}$ is very ample, then Castelnuovo's theorem, see [2] page 228, gives $c_{1}^{2}\left(Z_{i}\right) \geq 3 \chi\left(Z_{i}\right)-10$, which will contradict the above upper bound for $c_{1}^{2}\left(X_{i}\right)$. Thus, $Z_{i}$ must be chosen to have ample but not very ample canonical bundle, and will be in the sector where

$$
2 \chi\left(Z_{i}\right)-6 \leq c_{1}^{2}\left(Z_{i}\right)<3 \chi\left(Z_{i}\right),
$$

the first being the Noether inequality. The results of Xiao Gang and Z Chen, cf [14], show that all non-spin simply connected surfaces $Z_{i}$ with ample canonical bundle which are in this sector, and not too close to the line $c_{1}^{2}=3 \chi$, will have companions $X_{i}$ as required, obtained by blowing up minimal surfaces $Y_{i}$. Note that by Beauville's theorem on the canonical map, cf [2] page 228, all the $Z_{i}$ will be double covers of ruled surfaces.

We can avoid using the results of Xiao and Chen by taking for $Z_{i}$ the following family of Horikawa surfaces, of [2]. Let $\Sigma_{i}$ be the Hirzebruch surface whose section at infinity $S$ has self-intersection $-i$, and let $Z_{i}$ be a double cover of $\Sigma_{i}$ branched in a smooth curve homologous to $B=6 S+2(2 i+3) F$, where $F$ is the class of the fiber. The double cover is simply connected as $B$ is ample, and $K_{Z_{i}}=\pi^{*}\left(K_{\Sigma_{i}}+\frac{1}{2} B\right)=\pi^{*}(S+(i+1) F)$ is not 2 -divisible and so $Z_{i}$ is not spin. Moreover, $K_{Z_{i}}$ is the pullback of an ample line bundle and therefore ample, so that $Z_{i}$ admits a Kähler-Einstein metric. The characteristic numbers of $Z_{i}$ are $c_{1}^{2}\left(Z_{i}\right)=2 i+4$ and $\chi\left(Z_{i}\right)=i+5$.

Now, by the classical geography results of Persson [19], for all $i$ large enough there are simply connected surfaces $Y_{i}$ of general type with $c_{1}^{2}\left(Y_{i}\right)=6 i+13$ and $\chi\left(Y_{i}\right)=i+5$, so that the $(4 i+9)$-fold blowup $X_{i}$ of $Y_{i}$ is homeomorphic to $Z_{i}$.

The pairs $\left(X_{i}, Z_{i}\right)$ have all the desired properties.

Remark 1.4 The examples of manifolds without Einstein metrics given by LeBrun [18], namely blowups of hypersurfaces in $\mathbb{C} P^{3}$, cannot be used to prove Theorem 1.3 because they violate the Noether inequality. They are therefore not homeomorphic to minimal surfaces for which the resolution of the Calabi conjecture gives existence of an Einstein metric.

In view of Theorem 1.3, one can ask how many smooth structures with Einstein metrics and how many without, a given topological manifold has. On the 
one hand, using for example the work of Fintushel-Stern, one can show that one has infinitely many choices for the smooth structures of the manifolds $Y_{i}$ in the proof of Theorem 1.3, which remain distinct under blowing up points. Thus, one has infinitely many smooth manifolds one can use for each $X_{i}$, not admitting any Einstein metrics. On the other hand, it is known that there are homeomorphic non-diffeomorphic minimal surfaces of general type, cf [10], page 410, and the references cited there. In fact, the number of distinct smooth structures among sets of homeomorphic minimal surfaces of general type can be arbitrarily large [20]. It is not hard to check that all the examples in [20] and [10] have ample canonical bundle, and therefore have Kähler-Einstein metrics of negative scalar curvature. However, all those examples have $c_{1}^{2}>3 \chi$, and can therefore not be used as the $Z_{i}$ in the proof of Theorem 1.3. Still, those examples show that a given simply connected topological manifold can have an arbitrarily large number of smooth structures admitting Einstein metrics. Compare Theorem 2.2 below.

\section{Uniqueness for a given smooth structure}

We have seen that existence of Einstein metrics on closed 4-manifolds depends in an essential way on the smooth structure. I believe that the issue of uniqueness, up to the sign of the scalar curvature, is also tied to the smooth structure. More specifically:

Conjecture 2.1 A closed smooth 4-manifold admits Einstein metrics for at most one sign of the scalar curvature.

Such questions were raised in [3], pages 18-19, and are also addressed in [4]. What is new here, and in [4], is that the answer depends on the smooth structure, and also seems to depend on the dimension. The conjecture is interesting because it is sharp - it would be false if one did not fix the smooth structure, but only the underlying topological manifold:

Theorem 2.2 There are simply connected homeomorphic but non-diffeomorphic smooth 4-manifolds $X$ and $Y$, such that $X$ admits an Einstein metric of positive scalar curvature, and $Y$ admits an Einstein metric of negative scalar curvature.

Proof We can take for $X$ the 8 -fold blowup of $\mathbb{C} P^{2}$. By the work of TianYau [22] this admits a Kähler-Einstein metric of positive scalar curvature.

Geometry and Topology, Volume 2 (1998) 
For $Y$ we take the simply connected numerical Godeaux surface constructed by Craighero-Gattazzo [5] and studied recently by Dolgachev-Werner [6] ${ }^{2}$. This has ample canonical bundle, so that by the work of Aubin and Yau it admits a Kähler-Einstein metric of negative scalar curvature.

By Freedman's classification [9], $X$ and $Y$ are homeomorphic. That they are not diffeomorphic is clear from [13]. The argument carried out there for the Barlow surface, cf [1], works even more easily for the Craighero-Gattazzo surface as there is no complication arising from $(-2)$-curves. Alternatively, the fact that $X$ and $Y$ have Kähler-Einstein metrics of opposite signs implies via Seiberg-Witten theory that they are non-diffeomorphic.

In higher dimensions, homeomorphic non-diffeomorphic manifolds with Einstein metrics are known $[17,23]$, though in those examples all the metrics have positive scalar curvature. Although the examples of $[17,23]$ are consistent with a higher-dimensional analogue of the above conjecture, such a generalisation is false:

Corollary 2.3 For every $i \geq 2$ there is a simply connected closed $4 i$-manifold which admits Einstein metrics of both positive and negative scalar curvature.

Proof Let $X$ and $Y$ be as in Theorem 2.2. Then the $i$-fold products $X^{i}=$ $X \times \ldots \times X$ and $Y^{i}=Y \times \ldots \times Y$ have Kähler-Einstein metrics of positive, respectively negative, scalar curvature. However, as $X$ and $Y$ are simply connected and homeomorphic, they are $h$-cobordant. Therefore, $X^{i}$ and $Y^{i}$ are also $h$-cobordant for all $i$, and for $i \geq 2$ are diffeomorphic by the $h$-cobordism theorem.

Theorem 2.2 and Corollary 2.3 have also been proved independently by Catanese and LeBrun [4]. Instead of the Craighero-Gattazzo surface they use the Barlow surface, showing that it has deformations with ample canonical bundle. Conjecturally, the Barlow and Craighero-Gattazzo surfaces are deformation equivalent, and therefore diffeomorphic.

Acknowledgement: I am grateful to the Department of Mathematics at Brown University and to the Max-Planck-Institut für Mathematik in Bonn for hospitality and support.

\footnotetext{
${ }^{2}$ I am grateful to Igor Dolgachev for telling me about this, and for providing an advance copy of $[6]$.
}

Geometry and Topology, Volume 2 (1998) 


\section{References}

[1] $\mathbf{R}$ Barlow, A simply connected surface of general type with $p_{g}=0$, Invent. Math. 79 (1985) 293-301

[2] W Barth, C Peters, A Van de Ven, Compact Complex Surfaces, SpringerVerlag (1984)

[3] A L Besse, Einstein Manifolds, Springer-Verlag (1987)

[4] F Catanese, C LeBrun, On the scalar curvature of Einstein manifolds, preprint (May 1997)

[5] P Craighero, R Gattazzo, Quintic surfaces of $\mathbb{C} P^{3}$ having a nonsingular model with $q=p_{g}=0, P_{2} \neq 0$, Rend. Sem. Mat. Univ. Padova 91 (1994) $187-198$

[6] I Dolgachev, C Werner, A simply connected numerical Godeaux surface with ample canonical class, preprint (April 1997)

[7] SK Donaldson, The Seiberg-Witten equations and 4-manifold topology, Bull. Amer. Math. Soc. 33 (1996) 45-70

[8] R Fintushel, R J Stern, Immersed spheres in 4-manifolds and the immersed Thom conjecture, Proc. of Gökova Geometry-Topology Conference 1994, Turkish J. of Math. 19 (2) (1995) 27-39

[9] M H Freedman, The topology of four-manifolds, J. Differential Geometry 17 (1982) 357-454

[10] R Friedman, J W Morgan, Smooth Four-Manifolds and Complex Surfaces, Springer-Verlag (1994)

[11] M Gromov, Volume and bounded cohomology, Publ. Math. I.H.E.S. 56 (1982) 5-99

[12] N J Hitchin, Compact four-dimensional Einstein manifolds, J. Differential Geometry 9 (1974) 435-441

[13] D Kotschick, On manifolds homeomorphic to $\mathbb{C} P^{2} \# 8 \overline{\mathbb{C} P^{2}}$, Invent. math. 95 (1989) 591-600

[14] D Kotschick, Orientation-reversing homeomorphisms in surface geography, Math. Annalen 292 (1992) 375-381

[15] D Kotschick, P B Kronheimer, T S Mrowka, monograph in preparation

[16] D Kotschick, J W Morgan, C H Taubes, Four-manifolds without symplectic structures but with non-trivial Seiberg-Witten invariants, Math. Research Letters 2 (1995) 119-124

[17] M Kreck, S Stolz, A diffeomorphism classification of 7-dimensional homogeneous Einstein manifolds with $S U(3) \times S U(2) \times U(1)$ symmetry, Ann. of Math. 127 (1988) 373-388

[18] C LeBrun, Four-manifolds without Einstein metrics, Math. Research Letters 3 (1996) 133-147 
[19] U Persson, Chern invariants of surfaces of general type, Comp. Math. 43 (1981) 3-58

[20] M Salvetti, On the number of non-equivalent differentiable structures on 4manifolds, Manuscr. Math. 63 (1989) 157-171

[21] A Sambusetti, An obstruction to the existence of Einstein metrics on 4manifolds, C. R. Acad. Sci. Paris 322 (1996) 1213-1218

[22] G Tian, S-T Yau, Kähler-Einstein metrics on complex surfaces with $c_{1}>0$, Comm. Math. Phys. 112 (1987) 175-203

[23] McK Y Wang, W Ziller, Einstein metrics on principal torus bundles, J. Differential Geometry 31 (1990) 215-248

[24] E Witten, Monopoles and four-manifolds, Math. Research Letters 1 (1994) 769-796 